\title{
Liberia ebolafrei
}

Es scheint, als sei Westafrika bei der Bekämpfung der Ebolaepidemie fast anderthalb Jahre nach dem Auftreten der ersten Fälle endlich auf der Zielgeraden angekommen: Am 9. Mai - 42 Tage nach der Beerdigung des letzten Opfers - wurde zunächst Liberia offiziell als frei von Ebola erklärt. Und auch die anderen beiden noch betroffenen Länder verzeichneten in den vergangenen Wochen und Monaten erhebliche Erfolge. So wurden in Sierra Leone Ende Mai innerhalb einer Woche nur noch 3 Neuinfektionen bestätigt, Guinea meldete 9 Fälle.

Vermutlich existieren noch unbekannte Infektionsketten

Allerdings gibt es Indizien dafür, dass es immer noch unbekannte Infektionsketten in der Bevölkerung gibt und nicht alle Infizierten medizinische Hilfe suchen können oder wollen. So war etwa ein Drittel der im Mai erkrankten Personen den Behörden nicht als Kontakt eines frü- heren Ebolapatienten bekannt. Mehrere Infektionen wurden gar erst posthum bei Personen nachgewiesen, die zu Hause im Kreis ihrer Familie verstorben waren. Und immer noch gibt es Zwischenfälle, bei denen Aufklärungsteams oder medizinisches Personal von Menschen angegriffen werden, die der Überzeugung sind, Ebola gäbe es nicht oder die Krankheit werde erst durch die Helfer hervorgerufen.

Hinzu kommt, dass mittlerweile die Regenzeit eingesetzt hat und zahlreiche Dörfer von der medizinischen Versorgung und Beobachtung abgeschnitten sind. Sollte es auch in diesen abgelegenen Regionen noch unbekannte, aktive Virusträger geben, könnte sich die Krankheit hier womöglich einige Wochen lang ausbreiten, bevor die Zufahrtsstraßen wieder passierbar sind und Informationen hierüber die Außenwelt erreichen. Es bleibt zu hoffen, dass die Erfolge der letzten Wochen ausreichten, um die Epidemie einzudämmen und dies zu verhindern.
Bisher Tausende Tote in betroffenen Ländern

Insgesamt erkrankten seit Beginn der Epidemie im Dezember 2013 bisher etwa 27050 Menschen an Ebolainfektionen (dies beinhaltet alle registrierten Fälle ob bestätigt oder nicht - die Dunkelziffer ist vermutlich immens), mindestens 11150 hiervon überlebten die Infektion nicht. Sierra Leone meldete die meisten Verdachtsfälle (12706), gefolgt von Liberia (10666) und Guinea (3641). Die Zahl der registrierten Todesfälle war allerdings in Liberia mit 4806 am höchsten (Sierra Leone: 3908; Guinea: 2420). Bei etwa 870 der Infizierten handelte es sich um Krankenschwestern oder Ärzte, 507 von ihnen überlebten die Infektion nicht. Zuletzt erkrankte Anfang Mai ein freiwilliger medizinischer Helfer aus Italien nach seiner Rückkehr aus Sierra Leone.

Dr. Raymund Lösch und Dipl. Biol. Unn Klare, Bad Doberan

Quellen: promed, WHO, CDC

\section{Cholera auf Hispaniola}

Bereits seit Oktober 2010 leidet Haiti unter einer Choleraepidemie, bei der landesweit bisher mehr als 735000 Menschen erkrankten. Etwa 8760 von ihnen verstarben an den Folgen der Infektion. Aber auch 4,5 Jahre nach dem Beginn der Epidemie ist man noch weit davon entfernt, die Situation unter Kontrolle zu bringen. Im Gegenteil, momentan werden erneut steigende Fallzahlen gemeldet - so wurden allein in den ersten 3 Monaten dieses Jahres mehr als 10000 neue Verdachtsfälle registriert. Im Vergleichszeitraum des Vorjahrs waren es dagegen nur circa 3500. Nachdem vergangenes Jahr eine deutliche Reduktion der Fallzahlen erreicht werden konnte, ist man nun wieder auf dem Niveau des Jahres 2012 angekommen. Und mit dem Einsetzen der Regenzeit im Mai wird ein erneutes Ansteigen der Fallzahlen befürchtet.

Auch im Nachbarstaat, der Dominikanische Republik, bietet sich ein ähnliches Bild. Auch hier konnten im Jahr 2014 die
Fallzahlen deutlich gesenkt werden - so gab es $70 \%$ weniger registrierte Verdachtsfälle und sogar $76 \%$ weniger Todesopfer als noch 2013. In den ersten 10 Wochen dieses Jahres wurden dann aber mit 185 Verdachtsfällen wieder mehr als doppelt so viele Infektionen gemeldet wie im Vergleichszeitraum des Jahres 2014. Insgesamt stieg somit in der Dominikanischen Republik die Zahl der Verdachtsfälle seit Beginn der Epidemie im November 2010 auf 32250 (Stand Mitte März 2015). Mindestens 487 Menschen überlebten die Infektion nicht.

\section{Es fehlt sauberes Trinkwasser}

Vermutlich wird es Jahre dauern, bis diese Epidemie zu Ende gehen wird. Haiti ist in der westlichen Hemisphäre das Land mit der schlechtesten Trinkwasserversorgung - laut einem UN-Bericht aus dem vergangenen Jahr hatten 2013 mindestens 30\% der Bevölkerung keinen Zugang zu sauberem Trinkwasser. Andere Quellen gehen sogar davon aus, dass deutlich mehr als die Hälfte der Haitianer keine das ganze Jahr über verfügbare Quelle für sauberes Wasser besitzt. Selbst in Krankenhäusern ist die Versorgung mit sauberem Wasser nicht gesichert. Zugang zu funktionierenden sanitären Anlagen hat nur schätzungsweise jeder vierte Haitianer.

In der etwas wohlhabenderen Dominikanischen Republik ist die sanitäre Situation zwar besser, der Verlauf der Epidemie hier war aber in den vergangenen Jahren immer eng an den in Haiti gekoppelt. Ein Ende der Choleraepidemie in der Dominikanische Republik ist somit momentan nur realistisch, wenn es gelingt, auch die Fallzahlen in Haiti drastisch zu senken.

Dr. Raymund Lösch und Dipl. Biol. Unn Klare, Bad Doberan

Quellen: promed; paho; United Nations plan to support the implementation of the Government of Haiti's 2-year plan on cholera elimination, Januar 2014 


\section{Meningokokken im Niger}

Der Niger gehört zusammen mit 20 weiteren Ländern südlich der Sahara zum so genannten Meningitisgürtel Afrikas. Meningokokkeninfektionen treten hier regelmäßig auf und insbesondere in der Trockenzeit zwischen Dezember und Juni kommt es gelegentlich zu großen Epidemien mit Zehn- oder gar Hunderttausenden von Fällen. Der letzte große Ausbruch fand im Jahr 2009 statt, damals erkrankten vor allem im Niger und in Nigeria etwa 80000 Menschen. In der Vergangenheit wurden diese Ausbrüche fast ausschließlich durch den Erreger Neisseria meningitides Serotyp A hervorgerufen.

Keine Ausbrüche mehr durch Serotyp A Seit dem Jahr 2010 kommt nun ein neuer Konjugatimpfstoff (MenAfriVac) großflächig zum Einsatz, der extra für den afrikanischen Meningitisgürtel produziert wurde. MenAfriVac wirkt nur gegen den Serotyp A, ist sehr kostengünstig und extrem erfolgreich: In den Regionen, in denen MenAfriVac für Massenimmunisierungen verwendet wurde, gab es seither keine Meningokokken-A-Ausbrüche mehr.

Hohe Fallzahlen durch Serotyp C

Nun scheint es jedoch, als warte schon der nächste Erreger darauf, die Rolle von N. meningitides Serotyp A zu übernehmen. So erkrankten zwischen Anfang
Januar und Mitte Mai im Niger vermutlich 6180 Menschen an einer Meningokokken-Meningitis, die durch den Serotyp $C$ hervorgerufen wurde. Mindestens 423 Menschen verstarben hier an den Folgen der Infektion. Dabei nahm die Infektionswelle Anfang Mai erst so richtig Fahrt auf. Allein innerhalb der letzten 2 Wochen des Meldezeitraumes verdreifachten sich die Fallzahlen. Und auch Nigeria meldete bereits Anfang des Jahres einen Ausbruch von MeningokokkenMeningitis des Serotyps C. Bis Anfang April waren hier 1380 Verdachtsfälle (darunter 83 Todesopfer) registriert worden (aktuellere Zahlen liegen uns derzeit leider nicht vor).

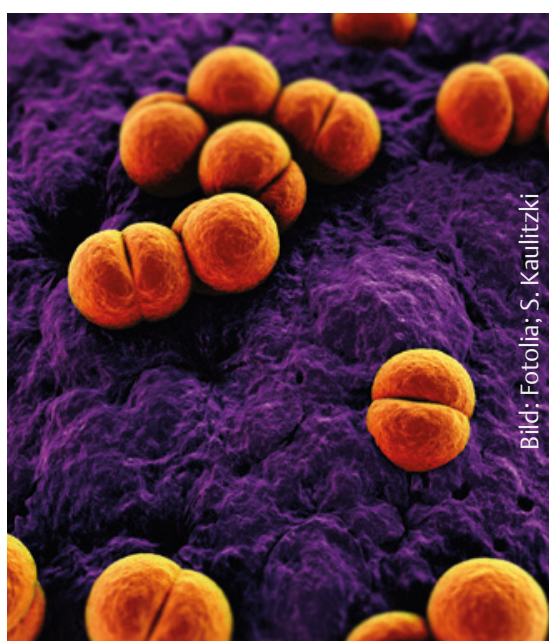

Dies ist das erste Mal, dass N. meningitides Serotyp C einen großen Ausbruch in Afrika verursacht. Obwohl dieser Serotyp in den wohlhabenderen Ländern der Welt der dominierende Meningokokkenerreger ist, gab es in Afrika in den vergangenen 40 Jahren nur sporadische Infektionen und einige wenige, kleinere Ausbrüche, die dann in der Regel durch eine Mischung von Serotyp-A- und Serotyp-C-Meningokokken verursacht wurden.

\section{Wenig Schutz gegen Serotyp C} vorhanden

Dadurch sind die afrikanischen Länder nicht ausreichend auf solch einen Ausbruch vorbereitet. In den vergangenen Jahren wurde hier ausschließlich gegen den Serotyp A geimpft. Ein Schutz gegen den derzeit dominierenden Serotyp $C$ besteht also beim Großteil der Bevölkerung nicht. Impfstoffe gegen andere als den Serotyp A sind nicht nur ausgesprochen knapp, in ganz Afrika handelt es sich dabei auch fast ausschließlich um Polysaccharidimpfstoffe, die keinen längerfristigen Schutz bieten. Die in Europa und den USA erhältlichen multivalenten Konjugatimpfstoffe sind für die betroffenen afrikanischen Länder unerschwinglich.

Dr. Raymund Lösch und Dipl. Biol. Unn Klare, Bad Doberan

Quelle: promed

\section{Ross-River-Virus in Australien}

Seit Beginn des Jahres erkrankten im australischen Bundesstaat Queensland etwa 3300 Menschen an Infektionen mit dem Ross-River-Virus. Dies ist der zweithöchste Wert der letzten 25 Jahre, im Durchschnitt werden bis Mitte März lediglich etwas mehr als 400 Fälle registriert.
Der Bundesstaat New South Wales meldete ebenfalls einen größeren Ausbruch: Hier infizierten sich seit dem Start der Regenzeit im Herbst 2014 bis Ende Januar 320 Menschen - etwa doppelt so viele wie ein Jahr zuvor. Und selbst aus Australiens Hauptstadt Canberra wurden min- destens 3 Fälle gemeldet. Diese Patienten hatten sich vor dem Auftreten der ersten Symptome jedoch auch in anderen Bundesstaaten aufgehalten, sodass nicht sicher ist, dass die Infektionen auch tatsächlich in Canberra erfolgten.

Das Ross-River-Virus wird durch Mücken übertragen, die normalerweise von September bis Mai insbesondere in der Zeit $\because$ des Sonnenuntergangs und den darauf folgenden 3 Stunden aktiv sind.

Dr. Raymund Lösch und Dipl. Biol. Unn Klare, Bad Doberan

Quelle: promed 
Nipah-Virus in Neukaledonien

\section{nachgewiesen}

Das zur Familie der Paramyxoviridae gehörende Nipah-Virus wurde erst im Jahre 1999 während eines Enzephalitisausbruchs unter Schweinebauern in Malaysia und Singapur entdeckt. Damals erkrankten fast 300 Menschen, mehr als 100 von ihnen überlebten die Infektion nicht. In Schweinen rief das Virus dagegen nur relativ leichte Krankheitssymptome hervor. Nach diesem einen Ausbruch wurden weder in Malaysia noch in Singapur jemals wieder weitere Fälle nachgewiesen - nicht in Menschen und auch nicht in Schweinen.

Allerdings melden seit dem Jahr 2001 nun Bangladesch und Singapur immer wieder Nipah-Virus-Infektionen. Hier erfolgt die Infektion jedoch in der Regel nicht durch erkrankte Schweine sondern durch Kontakt zu Flughunden und deren Ausscheidungen. Insbesondere mit Flughundurin oder -speichel kontaminierter Dattelpalmensaft stellt in Bangladesch eine häufige Infektionsquelle dar (es gibt jedoch auch Mensch-zuMensch-Übertragungen).

Das Reservoir der Nipah-Viren wird in eben diesen Flughunden der Gattung Pteropus vermutet. Das Verbreitungsgebiet der Viren ist nicht sicher bekannt. Zusätzlich zu den 4 Ländern, in denen bereits humane Fälle aufgetreten sind, konnte das Nipah-Virus in den vergangenen Jahren in thailändischen Flughunden direkt nachgewiesen werden. Darüber hinaus wurden Antikörper gegen Henipaviren (hierzu zählen neben NipahViren auch die nah verwandten HendraViren) in Flughunden aus dem gesamten Südostasiatischen Raum bis hin nach Madagaskar gefunden.

Anfang Mai dieses Jahres wurden nun erstmals in Neukaledonien mehrere Flughunde positiv auf Nipah-Viren getestet. Humane Fälle traten hier bisher nicht auf. Unserem Wissen nach ist dies der erste Nachweis von Nipah-Viren auf einer pazifischen Insel. Leider liegen derzeit keine Informationen darüber vor, mit welchem Test der Nachweis erfolgt ist.

Dr. Raymund Lösch und Dipl. Biol. Unn Klare, Bad Doberan

Quellen: promed, CDC

\section{Medizin an Bord: Was ist praktikabel?}

Schiffsärzte treffen je nach Schiffstyp auf unterschiedliche medizinische Ausrüstung und personelle Gegebenheiten. Das Hospital eines modernen Kreuzfahrtschiffs mit ausgebildetem Pflegepersonal und eventuell weiteren Kollegen eröffnet andere Möglichkeiten der Diagnostik und Therapie als der von einem Verein betriebene Traditionssegler, bei dem der Arzt im Bedarfsfall eher per „Hand gegen Koje“ mit seiner privaten Ausrüstung an Bord geht.

Dennoch unterliegen auch die teilweise in Hochglanzprospekten beworbenen Bordhospitäler moderner Kreuzfahrtschiffe mit Ultraschall- und Röntgengerät sowie OP und Intensiveinheit Restriktionen. Sowohl Kollegen anderer Fächer für konsiliarische Rückfragen als auch die Klinik der Maximalversorgung sind häufig nur eingeschränkt zugänglich. Die Evakuierung eines Patienten mit einem Helikopter ist - entgegen landläufiger Meinung - aufgrund großer Distanzen oder ungünstigem Wetter oft schwer oder gar nicht möglich.

Um gegen diese Widrigkeiten besser gewappnet zu sein, richtet die Kieler Schiffsarztlehrgang GbR nun bereits im vierten Jahr ihren Schiffsarztlehrgang Advanced Course aus. Ziel dieses Moduls ist es, den Teilnehmern durch Kollegen, die sowohl in ihren Fachgebieten als auch in der Medizin auf See erfahren sind, 2 Hauptaspekte zu vermitteln: Zum einen sollen sie erfahren, wie sie auch mit eingeschränkten diagnostischen und therapeutischen Möglichkeiten das Optimum für ihre Patienten erreichen können. Dazu erhalten sie Praxistipps, die speziell auf die Gegebenheiten an Bord zugeschnitten sind. Zum anderen soll der Umgang mit einem Dilemma diskutiert und damit erleichtert werden: Wann muss ein Patient von Bord, um ihn nicht zu gefährden, und wann kann er bleiben? Ein zu zögerliches Handeln, das zu Umrouting oder zu unnötigen Verzöge- rungen durch Helikoptereinsatz führen kann, wirkt sich erheblich auf den Schiffsbetrieb und den Reiseverlauf aus. Andererseits ist auch eine Gefährdung von Patienten indiskutabel, die daraus resultiert, dass der Schiffsarzt sich selbst und die Möglichkeiten an Bord überschätzt und aus diesem Grund auf eine Evakuierung verzichtet. Hierbei soll der Kurs die Sicherheit in der Entscheidungsfindung erhöhen.

Die Programmpunkte umfassen eine große Variationsbreite medizinischer Fächer von A wie Augenheilkunde bis Z wie Zahnmedizin. Dabei wird großer Wert auf eine Mischung aus theoretischen und praktischen Anteilen gelegt, sodass nicht nur Bekanntes wie das Nähen von Wunden oder die Darstellung freier Flüssigkeit im Notfallultraschall wiederholt werden, sondern auch für den Arzt an Land doch eher ungewöhnliche Themen wie die Gewinnung von Trinkwasserproben oder die Anwendung von Alkohol- und Drogentests auf dem Programm stehen.

Der Lehrgang findet in Kiel mit Blick auf die Kieler Förde und die Einfahrt des Nord-Ostsee-Kanals (der meistbefahrenen künstlichen Wasserstraße der Welt) statt. Die Ärztekammer SchleswigHolstein bewertet die Veranstaltung mit 60 Fortbildungspunkten.

Weitere Informationen sind im Internet unter www.schiffsarztlehrgang.de zu finden.

Quelle: Schiffsarztlehrgang GbR, Kiel 